\title{
TURNING NUMBERS FOR PERIODIC ORBITS OF DISK HOMEOMORPHISMS
}

\author{
MICHAL MISIUREWICZ AND ANA RODRIGUES
}

\begin{abstract}
We study braid types of periodic orbits of orientation preserving disk homeomorphisms. If the orbit has period $n$, we consider the closure of the $n$ th power of the corresponding braid and call linking numbers of the pairs of its components turning numbers. They are easy to compute and turn out to be very useful in the problem of classification of braid types, especially for small $n$.
\end{abstract}

\section{INTRODUCTION}

We want to study types of periodic orbits of orientation preserving disk homeomorphisms. This leads in a natural way to studying certain kinds of braids.

Let $f$ and $g$ be orientation preserving disk homeomorphisms with periodic orbits $P$ and $Q$ respectively (for simplicity, we will consider only orbits contained in the interior of the disk). Two such pairs $(f, P)$ and $(g, Q)$ are equivalent if $f$ is conjugate (in the dynamical systems meaning) to some $\widetilde{g}$ via a homeomorphism that maps $P$ to $Q$ and $\widetilde{g}$ is isotopic to $g$ relative to $Q$ (that is, via an isotopy that fixes the points of $Q)$. Equivalence classes are called braid types ([5]) or patterns ([11], [12]).

Thus, basically we are looking at the mapping classes of the homeomorphism relative to the periodic orbit. There is a well known connection with braids, explaining the name "braid types." Take the suspension flow of the homeomorphism with a periodic orbit $P$ and then the trajectory of any point $x \in P$ (cut at the level 0 ) can be identified with a braid. Since we pass from a 3-dimensional picture, where the points of the orbit are in the interior of the disk, to a basically 2-dimensional one, where they are ordered on an interval, the braid corresponding to an orbit is defined only up to an algebraic conjugacy (that is, the conjugacy in the braid group; we will refer to it just as conjugacy). Therefore, in order to study periodic orbits of orientation preserving disk homeomorphisms, we need to get information about braids up to conjugacy. This, in turn, requires some effective invariants of braid conjugacies.

There is a forcing relation on braid types [5]. A braid type $A$ forces braid type $B$ if every orientation preserving disk homeomorphism exhibiting $A$ has to exhibit $B$. This relation is a partial ordering [5].

According to the Nielsen-Thurston classification [15], if $f$ is an orientation preserving disk homeomorphism and $P$ is a periodic orbit, then there is a homeomorphism

Date: March 12, 2011.

2000 Mathematics Subject Classification. Primary 37E15, secondary 20F36 and 57M27.

Key words and phrases. conjugacy invariants of braids, positive permutation braids, braid types of periodic orbits.

Research of AR was supported by FCT Grant BPD/36072/2007 and in part by Centro de Matemática da Universidade do Porto (CMUP) financed by FCT through the programmes POCTI and POSI, with Portuguese and European Community structural funds. 
$g$, isotopic to $f$ relative to $P$ (so in particular, $\left.g\right|_{P}=\left.f\right|_{P}$ ), which is one of the three types:

(a) finite order (we will call it twist),

(b) pseudo-Anosov,

(c) reducible.

When studying forcing, it is important to know what type our orbit is. If it is a twist, then it does not force anything except a fixed point (and itself). If it is reducible, the problem of forcing can be also reduced to the study of forcing by two simpler orbits. Therefore we need to relate invariants of conjugacy of braids to the above classification.

From this point of view one useful invariant is known: the exponent sum (or wraith) $\operatorname{es}(B)$ of $B$ (we give the definition later). Here we want to show how to employ another class of braid invariants, linking numbers of the components of closures of appropriate powers of the braid, to the same aim. In order to simplify terminology, we will call those numbers turning numbers. They turn out to be refinements of the exponent sum.

Because we are interested in periodic orbits, not just finite invariant sets, we will concentrate mainly on the braids with cyclic permutations (that is, braids that close to a knot). We will call them cyclic braids. In particular, we will study positive permutation cyclic braids, which are braid types for disk homeomorphisms obtained by "thickening" interval maps. This class includes horseshoe braids, studied extensively by A. de Carvalho and T. Hall (see, e.g., [6] and [7]). As an application of turning numbers we will show how they can help in the classification of positive permutation cyclic braids with a given number of strands. This translates automatically into classification of periodic orbits of a given period of disk homeomorphisms of "thickened interval maps" type.

In the sequel, we will assume that the reader knows the basic notions of the braid theory. For an exposition on braids see, for example, [2], [3] or [8].

In Section 2 we define turning numbers of a braid and show a simple way of computing them. In Section 3 we define extensions of braids by other braids and show how to compute their turning numbers. In Section 4 we relate turning numbers with braids that come from periodic orbits of interval maps. In Section 5 we investigate turning numbers for twist braids and connect our results with those of [14]. In Section 6 we apply our results to the problem of classification of positive permutation cyclic braids. For each number of strands it has to be done separately; here we show with the details what one gets for 7 strands. In Section 7 we investigate connections between the first and the second turning numbers. In Section 8 we give an example of two relatively simple braids which have the same turning numbers but are not conjugate.

Let us stress that our aim was to find simple invariants, which, even if they are not too powerful, are easily defined and easily computable, so they can be easily used for our long-term primary goal - investigation of periodic orbits.

\section{DEFinition AND BASIC PROPERTIES}

Let $B$ be a braid with $n$ strands (an element of the $n$-th braid group) and permutation $\tau$ (that is, the $i$-th strand joins $i$ in the bottom with $\tau(i)$ at the top; we assume 
that the strands go up - this is because of the suspension model). For each crossing of two strands we define its sign in a standard way: it is \pm 1 depending on whether the left strand goes over the right one or vice versa. To fix notation, assume that in the former case it is +1 , and in the latter case -1 . We also agree that a strand does not cross itself.

We will be using multiplication in the braid and permutation groups [8]. To avoid misunderstandings, let us fix the notation now. We will use the opposite order than for composition of functions. That is, if $\sigma$ and $\tau$ are permutations, then $(\sigma \tau)(i)=\tau(\sigma(i))$. Similarly, if $A$ and $B$ are braids, then $A B$ will be the braid which we get by following first the strands of the braid $A$, and then the strands of the braid $B$.

Let us assume now that $B$ is cyclic (that is, $\tau$ is a cyclic permutation). Since the braids with $n$ strands form a group, we can consider the braid $B^{n}$. Its permutation is the identity; in other words, $B^{n}$ is a pure braid. This means that if we close this braid, that is, connect $i$ at the bottom with $i$ at the top for each $i$, without any new crossings (see, e. g., [8]), we will get a link with $n$ components. For any two components, $i$-th and $i+k$-th (where adding is modulo $n$ ) there is a well defined linking number between them. It is equal to $1 / 2$ of the sum of signs of crossings of $m$-th and $m+k$-th strands of $B^{n}$. If we split $B^{n}$ into "levels," each of them $B$, then such a crossing at $j$-th level is a crossing between $m+j$-th and $m+j+k$-th strands of $B$. Therefore, our linking number is equal to

$$
\mathrm{TN}_{k}(B)=\frac{1}{2} \sum_{i=1}^{n} T_{k}(i),
$$

where $T_{k}(i)$ is the sum of signs of the crossings between the $i$-th and $\tau^{k}(i)$-th strands. Now we see that indeed $\operatorname{TN}_{k}(B)$ depends on $k$, but not on $m$. We will call it the $k$-th turning number of $B$.

It is well known that linking numbers, and therefore turning numbers, are integers. Moreover, they are invariants of conjugacy. To see it, notice that if $B$ and $C$ are conjugate, so are $B^{n}$ and $C^{n}$, and their closures are embedded into $\mathbb{R}^{3}$ in the same way.

If the braid $B$ is not cyclic, its permutation can be split into several cyclic ones, and accordingly $B$ can be split, too. For each of the parts we can use the preceding definition (in such a way we just ignore crossings between strands in different cyclic components) and thus we can use (2.1) as a definition. Observe that in such a way we can define $\mathrm{TN}_{k}(B)$ for every $k$. Of course, $\mathrm{TN}_{0}(B)=0$.

Lemma 2.1. If a braid $B$ is cyclic with $n$ strands, then the $k$-th and $(n-k)$-th turning numbers of $B$ are equal. Moreover, for every $i$ the $(i n+k)$-th and $k$-th turning numbers of $B$ are equal.

Proof. A crossing of the $i$-th and $\tau^{k}(i)$-th strands is also a crossing of the $\tau^{k}(i)$-th and $\tau^{n-k}\left(\tau^{k}(i)\right)$-th strands. This proves the first statement. The second one follows from the fact that $\tau^{i n+k}=\tau^{k}$.

The exponent sum (or wraith) es $(B)$ of $B$ is defined as the sum of signs of all crossings in $B$. 
Theorem 2.2. If a braid $B$ with $n$ strands is cyclic then we have

$$
\mathrm{es}(B)=\sum_{k=1}^{n-1} \mathrm{TN}_{k}(B) \text {. }
$$

Proof. If we take the sum of the numbers $T_{k}(i)$ over $k=1, \ldots, n-1$ and $i=1, \ldots, n$, we get $2 \mathrm{es}(B)$, because in the sum every crossing is counted twice (as the crossing of of the $i$-th and $\tau^{k}(i)$-th strands and as the crossing of the $\tau^{k}(i)$-th and $\tau^{n-k}\left(\tau^{k}(i)\right)$-th strands). However, this sum is also equal to $2 \sum_{k=1}^{n-1} \mathrm{TN}_{k}(B)$.

Theorem 2.3. If $B$ is a cyclic braid with $n$ strands then for each $k$ and $m$ we have $\mathrm{TN}_{k}\left(B^{m}\right)=m \mathrm{TN}_{k m}(B)$.

Proof. We can think of $B^{m}$ as $m$ copies of $B$ stacked upon one another, forming $m$ levels. Each crossing occurs on some level. The permutation of $B^{m}$ is $\tau^{m}$. Thus, counting the crossings occurring at a given level and contributing to $\mathrm{TN}_{k}\left(B^{m}\right)$ is the same as counting the crossings for $B$, contributing to $\mathrm{TN}_{k m}(B)$. There are $m$ levels, so we get $\mathrm{TN}_{k}\left(B^{m}\right)=m \mathrm{TN}_{k m}(B)$.

Note that when applying the above theorem, we can take $\mathrm{km}$ modulo $n$, since the $k$-th and $\ell$-th turning numbers are the same if $k-\ell$ is divisible by $n$.

Another interesting observation is that the turning numbers are equal to coefficients of the Laurent polynomials defined by Fiedler [10] (see [13] for further research on Fiedler's polynomials). Indeed, in Definition 6 of [10] the sum is over all crossings. Each crossing comes with the sign +1 or -1 (this is $w(q)$ from Definition 1 ). Then $n^{+}(q)$ from Definition 6 (which is the same as $\xi^{+}(q)$ ) tells us how many times we have to go around in the knot to get back to the same crossing, when we start with the strand that is below the other one. This is the same as counting how many times we have to apply permutation to get from the lower strand at this crossing to the upper one. For $n^{-}$it is the same, but with the upper to lower. Thus, the exponent of $x$ in Definition 6 corresponds to $n-2 k$, when we compute the $k$-th turning number in a braid with $n$ strands, and the coefficient (the sum of $w(q)$ over all such crossings $q$ ) is this turning number.

We would like to thank the referee of the first version of our paper for pointing out the connections between the formula (2.1), linking numbers, and Fiedler's polynomial.

\section{Extensions}

As we mentioned in the introduction, one of the possibilities in the Nielsen-Thurston classification of periodic orbits is that the orbit is reducible. In such a case the structure of the orbit (call it $P$ ) is as follows. Up to isotopy rel. $P$, one can find a system of disks that are permuted by the map and $P$ is contained in their union. In each disk the number of elements of $P$ is the same, and it is larger than 1 but smaller than the period of $P$ (in particular, this is impossible if the period of $P$ is a prime number). Then we can consider two orbits: the outer one (call it $R$ ) is obtained by collapsing each disk to a point; the inner one (call it $Q$ ) is obtained by looking at one disk and taking the smallest iterate of the homeomorphism that maps this disk back to itself. In such a case one calls $P$ an extension of $R$ by $Q$, and similarly, the braid type of $P$ is the extension of the braid type of $R$ by the braid type of $Q$. For more information, see [11] or [12]. 
Translating this idea to the language of braids, for braids $B$ and $C$, where $B$ is cyclic, we can produce an extension of $B$ by $C$ as follows (see Figure 1). We replace
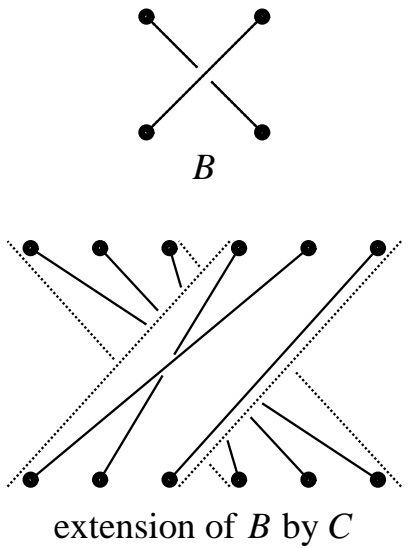

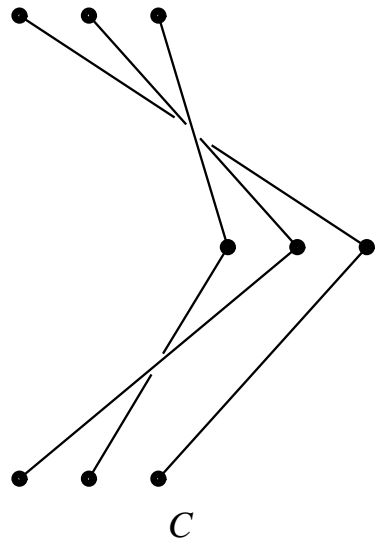

Figure 1. Construction of an extension.

each strand of $B$ by a wide "tape" and insert into each tape some braid (skewed, because the top and the bottom of the tape are disjoint). Each of the braids in the tapes has the same number of strands, so we can multiply them in the order given by the permutation of $B$. In other words, we follow the tape from the bottom to the top. then we jump vertically down, etc., until we get back to the place from which we started. The braid that we get this way should be $C$.

Note that we have some freedom in the construction, but all braids obtained as an extension of $B$ by $C$ are conjugate. Therefore we will use the name "extension of $B$ by $C$ " for any braid conjugate to the one obtained in the above construction.

Now we will show how to compute turning numbers of an extension of $B$ by $C$ from the turning numbers of $B$ and $C$.

Theorem 3.1. Let $B$ be a cyclic braid with $n$ strands, $C$ a braid with $r$ strands, and let $D$ be an extension of $B$ by $C$. If $k$ is divisible by $n$ then $\operatorname{TN}_{k}(D)=\operatorname{TN}_{k / n}(C)$. Otherwise, $\mathrm{TN}_{k}(D)=r \mathrm{TN}_{k}(B)$. Moreover, es $(D)=r^{2} \operatorname{es}(B)+\operatorname{es}(C)$.

Proof. We may assume that $D$ is the braid produced in the construction described earlier in this section. Consider first the case when $k$ is divisible by $n$. Then each crossing of strands number $i$ and $\tau^{k}(i)$, where $\tau$ is the permutation of $D$, occurs inside of a tape. Therefore it can be identified with the crossing of some strands number $j$ and $\eta^{k / n}(j)$ of $C$ (where $\eta$ is the permutation of $C$ ), with the same sign. This is a one-to-one correspondence, and therefore $\mathrm{TN}_{k}(D)=\mathrm{TN}_{k / n}(C)$.

Consider now the case when $k$ is not divisible by $n$. Then each crossing of strands number $i$ and $\tau^{k}(i)$ comes from a crossing of tapes, so it comes from the crossing of some strands number $j$ and $\zeta^{k}(j)$ of $B$ (where $\zeta$ is the permutation of $B$ ), with the same sign. There are $r$ strands of $D$ in a tape, so each such $j$ works for $r$ different numbers $i$. Therefore $\mathrm{TN}_{k}(D)=r \mathrm{TN}_{k}(B)$.

To compute es $(D)$, observe that counting with signs, there are $r^{2}$ es $(B)$ crossings of strands of $D$ that belong to different tapes, and es $(C)$ crossings of strands that belong to the same tape. 


\section{Connection With interval maps}

If $f$ is a continuous interval map with a periodic orbit $P$ then a homeomorphism of a "thick interval," which is homeomorphic to a disk, can be associated to it. Let us call the corresponding periodic orbit of this homeomorphism $P^{\prime}$. The interval defines the natural ordering on $P$, so when considering a braid associated to $P^{\prime}$, we have a natural choice of a braid. This braid is a positive permutation braid ([9], [14]), that is a braid with all crossings positive and each pair of strands crossing at most once. Its permutation (which is the same as the permutation of the points of the orbit $P$ of the interval map) is cyclic. We will call those braids positive permutation cyclic $(p p c)$ braids. Note that a positive permutation braid is uniquely determined by its permutation.

For a periodic orbit $P$ of an interval map $f$, draw its picture by putting an arrow for every $p \in P$ above the interval if $f(p)<p$, and below if $f(p)>p$ (see Figure 2; Figure 3 explains the connection with the graph of an interval map). This defines a piecewise smooth closed curve. When we go around it, note what the vector normal to the curve does (to make its movement continuous, smoothen the curve in a natural way). Denote the winding number of this vector around the origin by $m$.

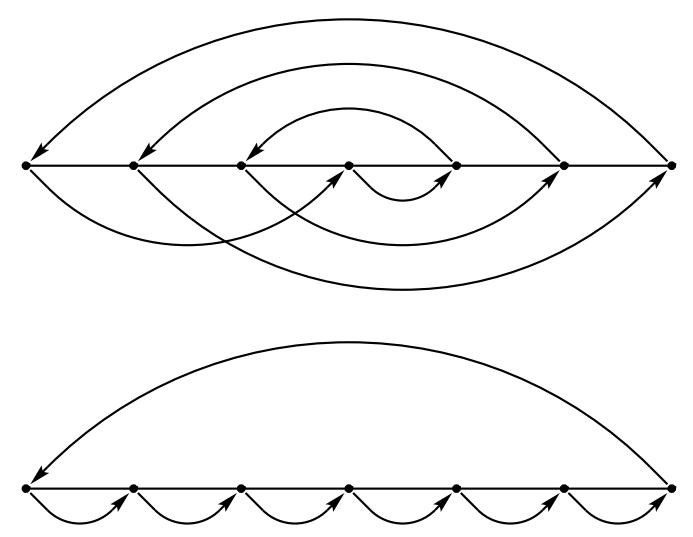

Figure 2. Two orbits of period 7 for interval maps.

It is clear that $m$ is equal to the number of times the arrow reverses its direction as we follow the curve, divided by 2 . Observe that the strands of the braid obtained from $(f, P)$ corresponding to $p$ and $f(p)$ intersect if and only if the directions of the arrows from $p$ to $f(p)$ and from $f(p)$ to $f^{2}(p)$ are opposite. This shows that the first turning number of this braid is $m$.

This fact motivates the name "turning number," since the first turning number just counts how many times the curve defined above turns around.

Let us make another interesting observation. This number, one half of the number of times the arrows from $p$ to $f(p)$ and from $f(p)$ to $f^{2}(p)$ have opposite directions as $p$ runs over $P$, is exactly the over-rotation number of $P$ [4].

For positive cyclic braids we can get more information about turning numbers, and even more is available for ppc braids. 


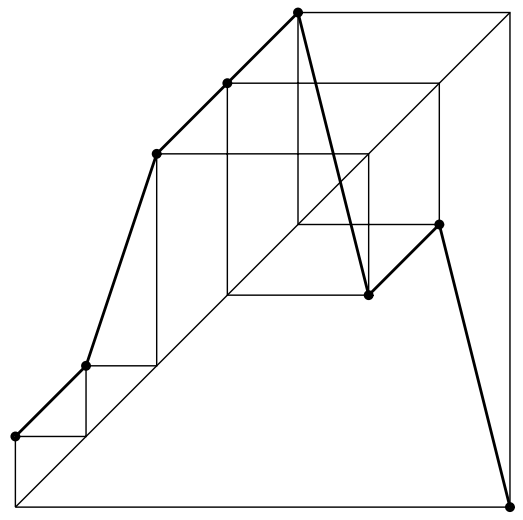

(a)

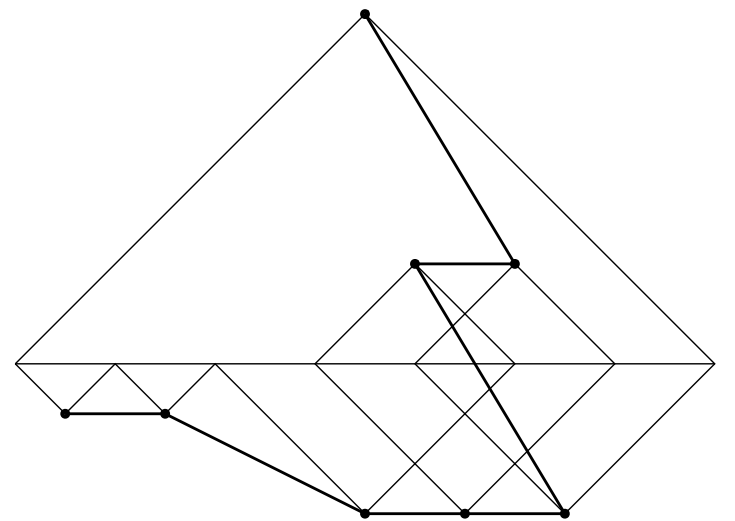

(b)

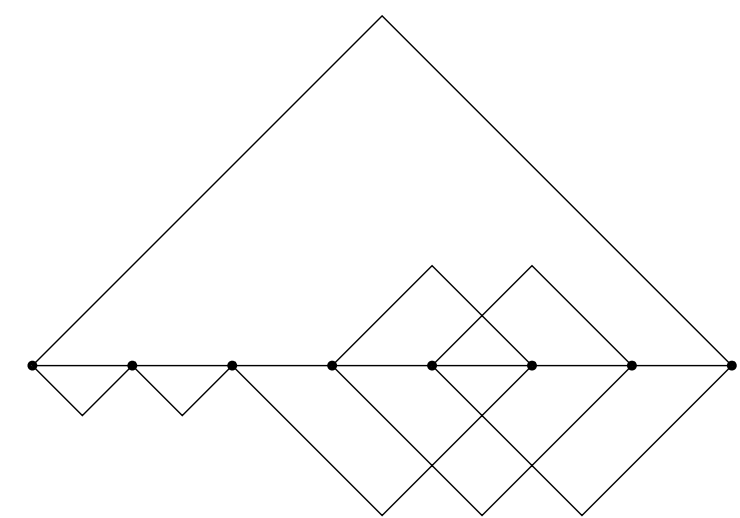

(c)

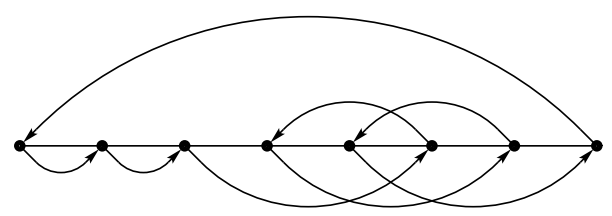

(d)

Figure 3. How to get a picture of an orbit from the graph of an interval map: (a) Draw the graph, mark the orbit and draw the usual cobweb for this orbit. (b) Apply the symmetry with respect to the diagonal and the clockwise rotation by 45 degrees. (c) Remove the graph and mark the points of the orbit on the (former) diagonal. (d) smoothen the curves joining marked points and make them arrows, upper ones pointing left and lower ones pointing right.

Lemma 4.1. Let $B$ be a braid with $n$ strands and cyclic permutation $\tau$. If strands number $j$ and $i$ do not cross then $j-i$ and $\tau(j)-\tau(i)$ have the same sign. If additionally we assume that $B$ is a ppc braid then we have equivalence: strands number $j$ and $i$ do not cross if and only if $j-i$ and $\tau(j)-\tau(i)$ have the same sign.

Proof. The first part is obvious. The second one follows from the first one and the fact that two strands in a ppc braid cross at most once.

Theorem 4.2. Let $B$ be a positive cyclic braid with $n$ strands. Then $\operatorname{TN}_{k}(B)$ is positive for $k=1,2, \ldots, n-1$. If additionally we assume that $B$ is a ppc braid then $\mathrm{TN}_{k}(B) \leq\lfloor n / 2\rfloor$.

Proof. Let $\tau$ be the permutation of $B$. Since $B$ is positive, $\mathrm{TN}_{k}(B) \geq 0$ for all $k$. By Lemma 4.1, if $T_{k}(i)=0$ then the signs of $i-\tau^{k}(i)$ and $\tau(i)-\tau^{k+1}(i)$ are the 
same. Since $\tau$ is cyclic, we get the $\operatorname{sign}$ of $j-\tau^{k}(j)$ the same for all $j$. However, for $k=1,2, \ldots, n-1$, if $j$ is the leftmost point, $\tau^{k}(j)$ is to its right and if $j$ is the rightmost point, $\tau^{k}(j)$ is to its left, a contradiction. Hence, $\mathrm{TN}_{k}(B)$ cannot be 0 .

If $B$ is a ppc braid then $T_{k}(i) \leq 1$ for all $i$, so we get $\operatorname{TN}_{k}(B) \leq n / 2$. However, $\mathrm{TN}_{k}(B)$ is an integer, so we get $\mathrm{TN}_{k}(B) \leq\lfloor n / 2\rfloor$.

\section{TWIST BRAIDS}

Let $F$ be the rotation of the unit disk by the angle $2 \pi m / n$, where $m$ and $n$ are coprime. Then all periodic orbits of $F$ in the interior of the disk (except its center) are twist orbits of period $n$. We call them $m / n$-twists, and the corresponding braids $m / n$ twist braids (for a given $m$ and $n$ there are many such braids, but by the definition, they are all conjugate). It is well known that in this way we get all braid types that correspond to the finite order maps in the Thurston's classification (it follows, for instance, from Theorem 12.5 of [11]).

The most natural $1 / n$-twist braid is the ppc braid with the permutation $\tau$ for which $\tau(i)=i+1$ for $i=1,2, \ldots, n-1$ and $\tau(n)=1$ (look at the second orbit at Figure 2). We will denote this braid by $B_{1 / n}$. Using the standard generators $\sigma_{1}, \ldots, \sigma_{n-1}$ of the $n$-th braid group, we can write $B_{1 / n}=\sigma_{n-1} \ldots \sigma_{2} \sigma_{1}$.

Composing disk maps sharing the same finite set as a periodic orbit (although the map on this orbit may be different) corresponds to the multiplication of braids. Thus, if $m$ and $n$ are coprime, the braid $B_{m / n}=B_{1 / n}^{m}$ is an $m / n$-twist braid. If $2 m<n$ (we always assume that $m$ is positive), then it is easy to see that it looks as follows. First $n-m$ strands go from $i$ to $i+m(i=1, \ldots, n-m)$ as straight segments. The last $m$ strands go from $i$ to $i+m-n$, but are twisted together by the full positive rotation (by 360 degrees; we do not want to use the word "twist" here, since we are using it with a slightly different meaning).

Let us conjugate this braid via the positive half-rotation (by 180 degrees) on the last $m$ strands. That is, we first apply the negative half-rotation on the last $m$ strands, then $B_{m / n}$, and then the positive half-rotation on the last $m$ strands. The first negative half-rotation will untwist partially the last $m$ strands, so there will be only one crossing between each pair of them. However, the positive half-rotation will twist the strands from $n-2 m+1$ through $n-m$, so again there will be one crossing between each pair of them. Thus, we can think of three bands of strands. The first one consists of $n-2 m$ strands and it goes in a monotone way (without crossings) by $m$ to the right (remember that the strands are going up). The second band consists of $m$ strands, the whole band goes to the right by $m$, but it is half-twisted. The third band consists also of $m$ strands and is half-twisted; the whole band goes by $n-m$ to the left. We denote this braid by $C_{m / n}$; it is a ppc braid. In Figure 4 we show the braids $C_{3 / 7}$ and $C_{1 / 7}$, corresponding to the twist orbits from Figure 2. Since each pair of strands crosses at most at one point, it is convenient to draw the strands as segments of straight lines (as we already did when defining extensions).

Let us compute the turning numbers of $B_{m / n}$. Since turning numbers are conjugacy invariants, they will be the same for all other $m / n$-twist braids.

Theorem 5.1. All turning numbers (from the first to $(n-1)$-st) of an $m / n$-twist braid are equal to $m$. 

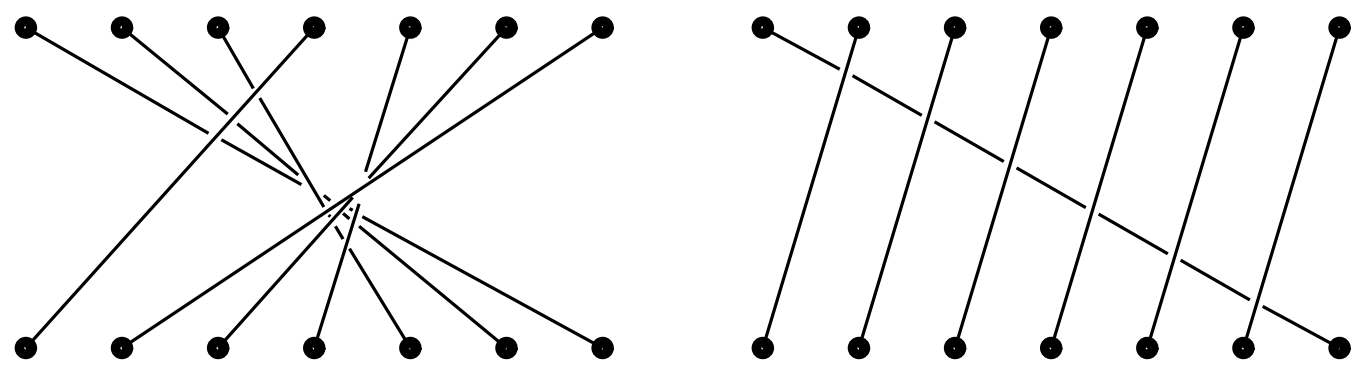

Figure 4 . Braids $C_{3 / 7}$ and $C_{1 / 7}$.

Proof. The structure of $B_{1 / n}$ is so simple, that the verification of $\mathrm{TN}_{k}\left(B_{1 / n}\right)=1$ for $k=1, \ldots, n-1$ is straightforward. Thus, by Theorem 2.3, we get $\operatorname{TN}_{k}\left(B_{m / n}\right)=m$. By the definition, each $m / n$-twist braid is conjugate to $B_{m / n}$, so since turning numbers are conjugacy invariants, they are equal to $m$.

Now we see that if $2 m>n$ then by Theorems 5.1 and $2.2 \operatorname{es}\left(B_{m / n}\right) \geq n(n-1) / 2$, so no $m / n$-twist braid can be a ppc braid.

Lemma 5.2. If a ppc braid with $n$ strands has the first turning number 1 , then its exponent sum is $n-1$.

Proof. Let $B$ be a ppc braid with $n$ strands and with the first turning number 1 . Let $\tau$ be its permutation. Then, by Lemma 4.1, there are only two numbers $i \in\{1, \ldots, n\}$ such that $\tau^{-1}(i)-i$ and $i-\tau(i)$ have opposite signs. Those $i$ 's have to be 1 and $n$. Therefore, there is $k<n$ such that

$$
1<\tau(1)<\tau^{2}(1)<\cdots<\tau^{k}(1)=n \text { and } n>\tau(n)>\tau^{2}(n)>\cdots>\tau^{n-k}(n)=1 .
$$

This means that in a picture like Figure 4 , over each gap between $j$-th and $(j+1)$-st points we see one strand going to the right and one going to the left. This proves that there are $n-1$ crossings, so es $(B)=n-1$.

Observe that since we are assuming here that the braid is cyclic, there must be a crossing in each gap as above, or, in other words, if we write a braid as a word in generators $\sigma_{i}$, each generator has to appear in this word.

Now we will use the results of the paper [14]. Since not everything that is proven there is stated as a theorem (some things important to us are hidden in the proofs of Theorems 2, 3 and 5), we will restate those results in a form useful to us. By $\Delta_{n}$ we denote the positive half-rotation on all $n$ strands.

Theorem 5.3 ([14]). Let $B$ be a ppc braid with $n$ strands. Then

(a) If $B$ closes to the unknot then $\operatorname{es}(B)=n-1$; if $B$ closes to a trefoil, then $\operatorname{es}(B)=n+1$.

(b) If es $(B)=n-1$ then $B$ is conjugate to $\sigma_{1} \sigma_{2} \ldots \sigma_{n-1}$.

(c) If es $(B)=n+1$ then $B$ is conjugate to $\sigma_{1}^{3} \sigma_{2} \ldots \sigma_{n-1}$.

(d) If $\operatorname{es}(B)=n(n-1) / 2-\lfloor(n-1) / 2\rfloor$ then $B$ is conjugate to the braid $E_{n}=$ $\Delta_{n} \sigma_{1}^{-1} \sigma_{2}^{-1} \ldots \sigma_{\lfloor(n-1) / 2\rfloor}^{-1}$ (see Figure 5$)$. 

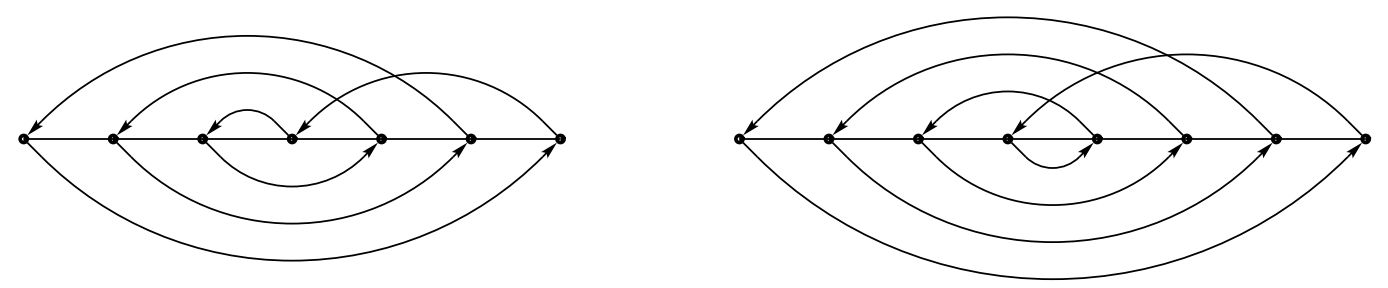

Figure 5. Braids $E_{7}$ and $E_{8}$.

Remark 5.4. In [14], it is stated without proof that the number from (d) above is the maximal possible exponent sum of a ppc braid. To see that a ppc braid with this exponent sum exists, one can just compute the exponent sum of $E_{n}$ (which we will do later).

However, one has to check that a larger exponent sum cannot occur. If $n$ is odd, this follows immediate from our Theorems 2.2 and 4.2. To give a general proof, note that a ppc braid can be written as $\Delta_{n} \sigma_{i_{1}}^{-1} \sigma_{i_{2}}^{-1} \ldots \sigma_{i_{r}}^{-1}$ (see [9]). The permutation of $\Delta_{n}$ consists of $\lfloor(n+1) / 2\rfloor$ cycles. Each $\sigma_{i_{j}}^{-1}$ can reduce the number of cycles at most by 1 . Thus, $r \geq\lfloor(n+1) / 2\rfloor-1=\lfloor(n-1) / 2\rfloor$, and therefore the exponential sum of our braid is at most $n(n-1) / 2-\lfloor(n-1) / 2\rfloor$.

Now we can strengthen statements (b)-(d) of Theorem 5.3.

Theorem 5.5. Let $B$ be a ppc braid with $n$ strands. Then the following conditions are equivalent:

(a) $\operatorname{es}(B)=n-1$

(b) $\mathrm{TN}_{1}(B)=1$,

(c) $\mathrm{TN}_{1}(B)=\mathrm{TN}_{2}(B)=\cdots=\mathrm{TN}_{n-1}(B)=1$,

(d) $B$ is a $1 / n$-twist braid.

Proof. $(\mathrm{a}) \Rightarrow(\mathrm{d})$. By Theorem $5.3(\mathrm{~b}), B$ is conjugate to $\sigma_{1} \sigma_{2} \ldots \sigma_{n-1}$. By Theorems 5.1 and $2.2, B_{1 / n}$ has exponent sum $n-1$, so by Theorem 5.3 (b) it is also conjugate to $\sigma_{1} \sigma_{2} \ldots \sigma_{n-1}$. Thus, $B$ is conjugate to $B_{1 / n}$, so it is a $1 / n$-twist braid.

$(\mathrm{d}) \Rightarrow(\mathrm{c})$. Follows from Theorem 5.1.

$(\mathrm{c}) \Rightarrow(\mathrm{b})$. Obvious.

(b) $\Rightarrow(\mathrm{a})$. This is Lemma 5.2 .

Theorem 5.6. Let $B$ be a ppc braid with $n$ strands. Then the following conditions are equivalent:

(a) $\operatorname{es}(B)=n+1$,

(b) $\mathrm{TN}_{1}(B)=\mathrm{TN}_{n-1}(B)=2$ and $\mathrm{TN}_{2}(B)=\cdots=\mathrm{TN}_{n-2}(B)=1$.

(c) $B$ is conjugate to $\sigma_{1}^{3} \sigma_{2} \ldots \sigma_{n-1}$.

Proof. (a) $\Rightarrow$ (c). This is Theorem 5.3 (c).

$(\mathrm{c}) \Rightarrow(\mathrm{b})$. By Theorem 5.1, $\mathrm{TN}_{1}\left(B_{1 / n}\right)=\mathrm{TN}_{2}\left(B_{1 / n}\right)=\cdots=\mathrm{TN}_{n-1}\left(B_{1 / n}\right)=1$. Compared to $B_{1 / n}$, we have in $\sigma_{1}^{3} \sigma_{2} \ldots \sigma_{n-1}$ two more crossings, between strands number 2 and $1=\tau(2)$ (where $\tau$ is the permutation of this braid). This adds 1 to the first and $(n-1)$-st turning numbers.

$(\mathrm{b}) \Rightarrow(\mathrm{a})$. Follows from Theorem 2.2 . 
Theorem 5.7. Let $B$ be a ppc braid with $n$ strands. If $n$ is odd then the following conditions are equivalent:

(a) $\operatorname{es}(B)=(n-1)^{2} / 2$,

(b) $\mathrm{TN}_{1}(B)=\mathrm{TN}_{2}(B)=\cdots=\mathrm{TN}_{n-1}(B)=(n-1) / 2$,

(c) $B$ is a $\frac{n-1}{2} / n$-twist braid.

If $n$ is even then the following conditions are equivalent:

(a') $\operatorname{es}(B)=\left((n-1)^{2}+1\right) / 2$,

(b') if $1 \leq k \leq n-1$ then $\mathrm{TN}_{k}(B)=n / 2$ for $k$ odd and $\mathrm{TN}_{k}(B)=n / 2-1$ for $k$ even.

(c') $B$ is an extension of $B_{1 / 2}$ by $B_{(n / 2-1) /(n / 2)}$.

Proof. Note first that if $n$ is odd then $n(n-1) / 2-\lfloor(n-1) / 2\rfloor=(n-1)^{2} / 2$, and if $n$ is even then $n(n-1) / 2-\lfloor(n-1) / 2\rfloor=\left((n-1)^{2}+1\right) / 2$.

$(\mathrm{a}) \Rightarrow(\mathrm{c})$. By Theorem $5.3(\mathrm{~d}), B$ is conjugate to $E_{n}$. By Theorems 5.1 and 2.2, $B_{\frac{n-1}{2} / n}$ has exponent sum $(n-1)^{2} / 2$, so by Theorem $5.3(\mathrm{~d})$ it is also conjugate to $E_{n}$. Thus, $B$ is conjugate to $B_{\frac{n-1}{2} / n}$, so it is a $\frac{n-1}{2} / n$-twist braid.

(c) $\Rightarrow(\mathrm{b})$. Follows from Theorem 5.1 .

(b) $\Rightarrow(\mathrm{a})$. Follows from Theorem 2.2.

Now we consider the case of $n$ even. Let us start with showing that $E_{n}$ is an extension of $B_{1 / 2}$ by $B_{(n / 2-1) /(n / 2)}$. Since $\Delta_{n}$ is an extension of $B_{1 / 2}$ by $\Delta_{n / 2}^{2}$, the braid $E_{n}$ is an extension of $B_{1 / 2}$ by the braid $D=\Delta_{n / 2}^{2} \sigma_{1}^{-1} \sigma_{2}^{-1} \ldots \sigma_{n / 2-1}^{-1}=\Delta_{n / 2}^{2} B_{1 /(n / 2)}^{-1}$ (cf. Figure 5). Since $\Delta_{n / 2}^{2}=B_{1 /(n / 2)}^{n / 2}$, we get $D=B_{1 /(n / 2)}^{n / 2-1}=B_{(n / 2-1) /(n / 2)}$. Thus, $E_{n}$ is an extension of $B_{1 / 2}$ by $B_{(n / 2-1) /(n / 2)}$.

$\left(\mathrm{a}^{\prime}\right) \Rightarrow\left(\mathrm{c}^{\prime}\right)$. In view of what we proved above, this is Theorem $5.3(\mathrm{~d})$.

$\left(c^{\prime}\right) \Rightarrow\left(b^{\prime}\right)$. Follows from Theorems 5.1, 2.2 and 3.1.

$\left(b^{\prime}\right) \Rightarrow\left(a^{\prime}\right)$. Follows from Theorem 2.2.

Motivated by Theorems 5.5 and 5.7, we state the following conjecture.

Conjecture 5.8. Let $m, n$ be positive coprime integers with $2 m<n$ and let $B$ be a ppc braid with $n$ strands and with $\mathrm{TN}_{1}(B)=\mathrm{TN}_{2}(B)=\cdots=\mathrm{TN}_{n-1}(B)=m$. Then $B$ is an $m / n$-twist braid.

\section{Applications}

Now we show how to apply the notion of turning numbers to the problem of classification of positive permutation cyclic braids. This translates automatically into classification of periodic orbits of a given period of disk homeomorphisms of "thickened interval maps" type. By "classification" one can understand two things. One is deciding whether a given braid is twist, reducible, or pseudo-Anosov in the NielsenThurston classification. The second thing is deciding whether two given braids are conjugate to each other.

It is clear how turning numbers can help in achieving those two goals. Theorem 5.1 tells us how the turning numbers of a twist braid look, and Theorem 3.1 does the same for reducible braids. If the turning numbers of a given braid are not of the form described in one of those theorems, we know immediately that the braid is pseudoAnosov. In particular, if the number $m$ of strands of a braid $D$ is a prime number, $D$ 
cannot be reducible, so if not all turning numbers of $D$ (from the first to $(m-1)$-st) are equal, then by Theorem $5.1 \mathrm{D}$ is pseudo-Anosov.

Using Theorem 3.1 is more difficult. We can state the precise way we do it as follows.

Proposition 6.1. If a ppc braid $D$ with $m$ strands is reducible, then there exists a proper divisor $n$ of $m$ such that $T N_{n}(D) \geq T N_{2 n}(D)$ and for all $k$ not divisible by $n$ the turning number $T N_{k}(D)$ is divisible by $\mathrm{m} / \mathrm{n}$.

Proof. If $D$ is an extension of a braid $B$ with $n$ strands by some braid $C$, then by Theorem 3.1, $T N_{n}(D)=T N_{1}(C)$ and $T N_{2 n}(D)=T N_{2}(C)$, so by Corollary 7.3 (which we will prove in the next section) $T N_{n}(D) \geq T N_{2 n}(D)$. Moreover, if $k$ is not divisible by $n$ then, by Theorem 3.1, $T N_{k}(D)$ must be divisible by $m / n$.

When we want to check whether two given braids are conjugate to each other, we compute their turning numbers and compare them. If they are different, the braids are not conjugate.

As an illustration to the use of turning numbers, we will present the classification of the ppc braids with 7 strands, where it gives remarkable results.

The standard way to decide whether there is a conjugacy between two ppc braids $B$ and $C$ with $n$ strands uses the theorem saying that they are conjugate if and only if there exist ppc braids $B_{0}=B, B_{1}, \ldots, B_{p-1}, B_{p}=C$ such that $B_{i}$ and $B_{i+1}$ are conjugate via a positive permutation braid (see, e.g., Corollary 4.2 of [9]). This requires multiple conjugations of various braids via all positive permutation braids (there are $n$ ! of them). Instead, we can use a much simpler equivalence relation, where $B_{i}$ and $B_{i+1}$ are conjugate via a simple switch (a generator braid $\sigma_{j}$ ); there are only $n-1$ switches. Then we use turning numbers to compare braids. While in a general case it does not have to work (as we show in Section 8), quite often it does.

Using the simple equivalence relation described above, we get for $n=7$ fifteen different equivalence classes, whose representatives are labeled $\mathrm{A}-\mathrm{O}$ in Figure 6 . The first 3 turning numbers are also displayed there (by Lemma 2.1 the next 3 turning numbers are the same, in the reverse order). Now we see that in this list there are only 3 candidates for twist braids, so they must be twist: $\mathrm{A}$ is a $1 / 7$-twist, $\mathrm{E}$ is a $2 / 7$-twist, and $\mathrm{K}$ is a $3 / 7$-twist. Since 7 is a prime number, all other braids from Figure 6 must be pseudo-Anosov. Moreover, all those braids have distinct turning numbers, so they are pairwise non-conjugate. In this way, we know that the set of all ppc braids with 7 strands is partitioned into 15 conjugacy classes, 3 of them twist and 12 pseudo-Anosov. We know a representative of each conjugacy class and if we want, we can easily draw all 720 ppc braids with 7 strands, indicating to which conjugacy class each of them belongs.

\section{FIRST AND SECOND TURNING NUMBERS}

In this section we will explain how to compute easily the first and second turning numbers for the ppc braids and how to compare them. Let $B$ be a ppc braid with $n>2$ strands and permutation $\tau$. We can think of $\tau$ as walking on $\{1,2, \ldots, n\}$, sometimes to the right, sometimes to the left, and with varying step length. We compare two first steps we make from $i \in\{1,2, \ldots, n\}$. If we move in the same direction, that is $\tau(i)-i$ and $\tau^{2}(i)-\tau(i)$ have the same signs, we assign to $i$ the 


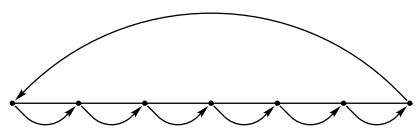

A 111

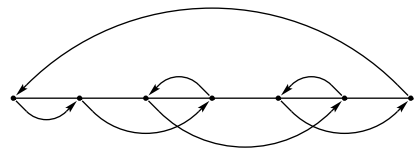

D 311

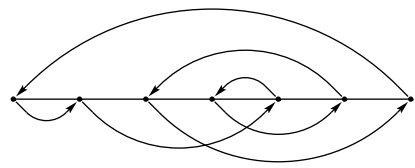

G 322

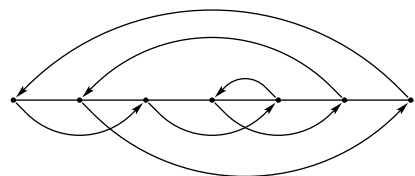

J 323

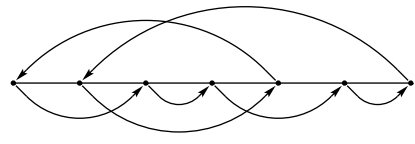

M 212

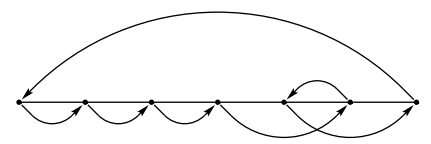

B 211

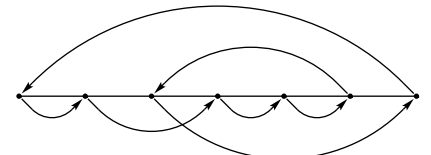

E 222

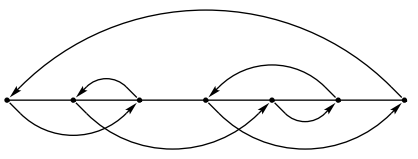

H 321

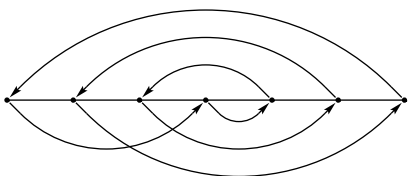

K 333

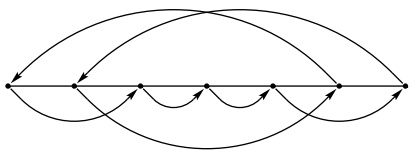

N 213

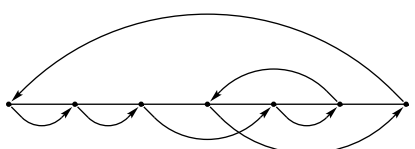

C 221

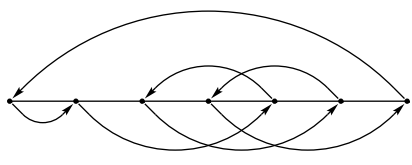

F 312

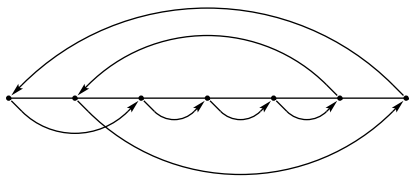

I 223

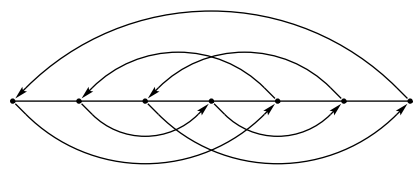

L 332

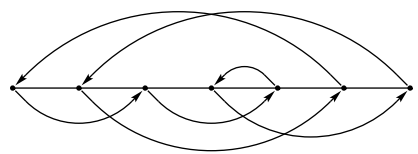

O 313

Figure 6. Positive permutation braids of period 7, up to conjugacy.

symbol $o$. If we move in opposite directions, that is $\tau(i)-i$ and $\tau^{2}(i)-\tau(i)$ have opposite signs, we compare the lengths of those two steps. If the second step is shorter than the first one, that is, $\left|\tau^{2}(i)-\tau(i)\right|<|\tau(i)-i|$, then we assign to $i$ the symbol $s$. If the second step is longer than the first one, that is, $\left|\tau^{2}(i)-\tau(i)\right|>|\tau(i)-i|$, then we assign to $i$ the symbol $\ell$. Now, when we follow our $\tau$-orbit (that is, consider $\tau^{k}(1)$, $k=0,1, \ldots$, where $k$ is taken modulo $n$ ), we get a circular sequence of symbols $o, s, \ell$ of length $n$. We call this sequence the code of $\tau$ (or of $B$ ).

Theorem 7.1. The number of symbols $s$ and $\ell$ (together) in the code of a ppc braid $B$ is equal to $2 \mathrm{TN}_{1}(B)$.

Proof. Symbols $s$ and $\ell$ are assigned exactly to those $i$ 's for which there is a crossing between the $i$-th and $\tau(i)$-th strand.

Theorem 7.2. The number of blocks sl in the code of a ppc braid $B$ is equal to $\mathrm{TN}_{1}(B)-T N_{2}(B)$.

Proof. Let us find out for which pairs of symbols assigned to $i$ and $\tau(i)$ the numbers $\tau^{3}(i)-\tau(i)$ and $\tau^{2}(i)-i$ have opposite signs. According to Lemma 4.1, those are the 
$i$ 's for which the strands number $i$ and $\tau^{2}(i)$ cross each other, so the number of those $i$ 's is equal to $2 T N_{2}(B)$. A direct inspection (see Figure 7) shows that this happens

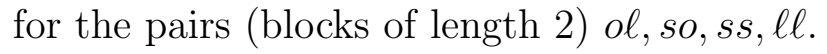

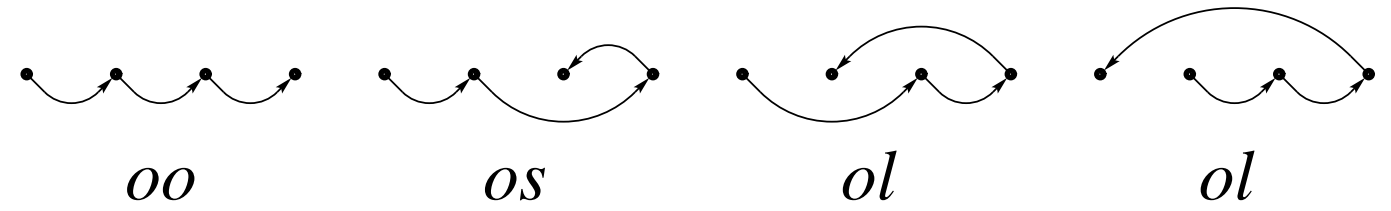

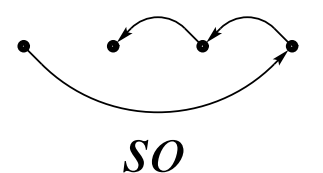

SO

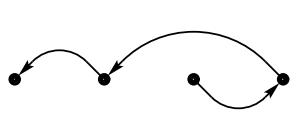

lo

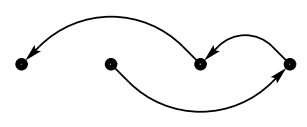

SO

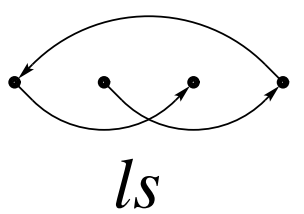

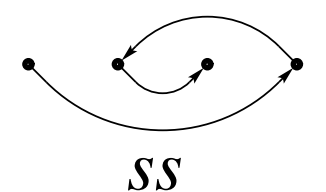

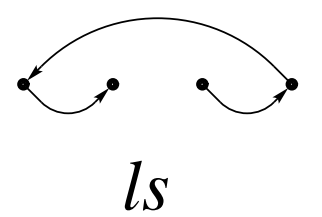

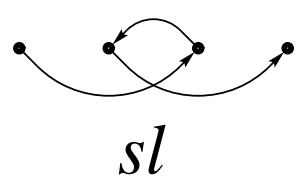

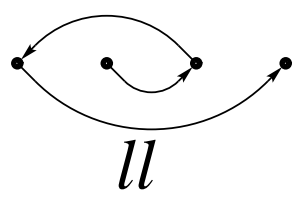

FiguRE 7 . Various blocks of length 2 in the code of $B$.

In other words, in order to compute $2 T N_{2}(B)$ we go around the code and count how many times we encounter blocks $o \ell, s o, s s, \ell$. Since these are all pairs whose first element is $s$ or the second element is $\ell$, except the pair $s \ell$, we have to add the number of $s$ 's and $\ell$ 's in the code and subtract twice the number of the blocks $s \ell$ in the code. Thus, in view of Theorem $7.1,2 T N_{2}(B)$ is equal to $2 \mathrm{TN}_{1}(B)$ minus twice the number of the blocks $s \ell$ in the code.

Corollary 7.3. If $B$ is a ppc braid then $T N_{2}(B) \leq T N_{1}(B)$. Moreover, the equality holds if and only if there are no blocks sl in the code of $B$.

\section{ExPerimental RESUlts}

As we saw in Section 6, for ppc braids with 7 strands, two braids with the same turning numbers are always conjugate. Computations show that the same holds for ppc braids with less than 7 strands. Unfortunately, there is a counterexample to this statement for braids with 8 strands. Both braids corresponding to the periodic orbits from Figure 8 have the same turning numbers: 3, 1,2,1,2,1,3, but they are not conjugate.

To see that the braids are not conjugate, one can find their pseudo-Anosov representations, using the methods of [1] or [11] (see also [12]). Corresponding Markov partitions give transition matrices that allow us to estimate their entropies. 

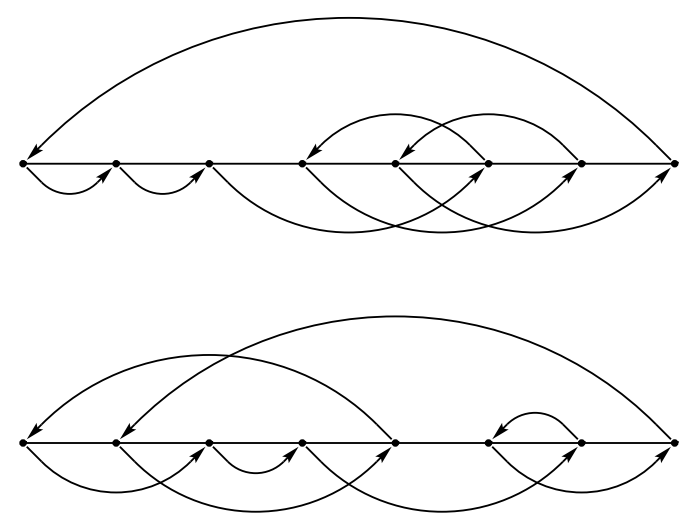

Figure 8 . Two orbits of period 8 with the same turning numbers but different braid types.

The first matrix is

$$
\left[\begin{array}{lllllll}
0 & 1 & 0 & 0 & 0 & 0 & 0 \\
0 & 0 & 1 & 1 & 1 & 1 & 0 \\
0 & 0 & 0 & 0 & 0 & 1 & 0 \\
0 & 0 & 0 & 0 & 0 & 1 & 1 \\
0 & 0 & 0 & 0 & 1 & 1 & 1 \\
0 & 0 & 0 & 1 & 0 & 0 & 0 \\
1 & 1 & 1 & 0 & 0 & 0 & 0
\end{array}\right]
$$

and the second matrix is

$$
\left[\begin{array}{lllllll}
0 & 1 & 1 & 0 & 0 & 0 & 0 \\
1 & 1 & 1 & 1 & 0 & 0 & 0 \\
0 & 0 & 0 & 0 & 1 & 1 & 0 \\
1 & 2 & 0 & 0 & 0 & 0 & 0 \\
2 & 2 & 0 & 1 & 1 & 1 & 1 \\
0 & 0 & 0 & 0 & 0 & 1 & 1 \\
1 & 1 & 0 & 1 & 1 & 0 & 0
\end{array}\right] .
$$

The cube of the second matrix has the minimal row sum 21, so the topological entropy of any orientation preserving disk homeomorphism exhibiting the second orbit is at least $\frac{1}{3} \log 21$. On the other hand, the cube of the first matrix has the maximal row sum 15 , so there is an orientation preserving disk homeomorphism exhibiting the second orbit with topological entropy less than or equal to $\frac{1}{3} \log 15$.

\section{REFERENCES}

[1] M. Bestvina and M. Handel, Train tracks for surface homeomorphisms, Topology 34, 109-140 (1995).

[2] J. S. Birman and T. E. Brendle, Braids: A survey, Handbook of knot theory. Elsevier. Amsterdam (2005).

[3] J. S.Birman, Braids, Links and Mapping Class Groups, Annals of Mathematics Studies. Princeton University Press. Princeton, NJ (1975).

[4] A. Blokh and M. Misiurewicz, New order for periodic orbits of interval maps, Ergod. Th. E Dynam. Sys. 17, 565-574 (1997).

[5] P. Boyland, Topological methods in surface dynamics, Topology Appl. 58, 223-298 (1994). 
[6] A. de Carvalho and T. Hall, The forcing relation for horseshoe braid types, Experimental Math. 11, 271-288 (2002).

[7] A. de Carvalho and T. Hall, Conjugacies between horseshoe braids, Nonlinearity 16, 1329-1338 (2003).

[8] M. Chiodo, An Introduction to Braid Theory, Msc Thesis, University of Melbourne (2005).

[9] E. A. Elrifai and H. R. Morton, Algorithms for positive braids, The Quarterly Journal of Mathematics 45, 479-497 (1994).

[10] T. Fiedler, A small state-sum invariant for knots, Topology 32, 281- 294 (1993).

[11] J. Franks and M. Misiurewicz, Cycles for disk homeomorphisms and thick trees, Contemporary Mathematics 152, 69-139 (1993).

[12] J. Franks and M. Misiurewicz, Topological methods in dynamics, Handbook of Dynamical Systems, Vol.1A 152, 547-598 (2002).

[13] H. R. Morton, The Burau matrix and Fiedler's invariant for a closed braid, Topology Appl. 95 251-256 (1999).

[14] H. R. Morton and R. J. Hadji, Conjugacy for positive permutation braids, Fundamenta Mathematicae 188, 155-166 (2005).

[15] W. Thurston, On the geometry and dynamics of diffeomorphisms of surfaces, Bull. Amer. Math. Soc. 19, 417-431 (1988).

Department of Mathematical Sciences, iUPui, 402 N. Blackford Street, IndiANAPOLIS, IN 46202-3216, USA

E-mail address: mmisiure@math.iupui.edu

Department of Mathematics, KTH, SE-100 44 Stockholm, Sweden

E-mail address: amdsar@kth.se 